\title{
Forces involved in bacterial adhesion to hydrophilic and hydrophobic surfaces
}

\author{
Correspondence \\ Henny C. van der Mei \\ h.c.van.der.mei@med.umcg.nl
}

Received 18 March 2008

Revised 24 June 2008

Accepted 27 June 2008

\author{
Niels P. Boks, ${ }^{1}$ Willem Norde, ${ }^{1,2}$ Henny C. van der Mei ${ }^{1}$ \\ and Henk J. Busscher ${ }^{1}$ \\ ${ }^{1}$ Department of Biomedical Engineering, University Medical Center Groningen and University of \\ Groningen, Antonius Deusinglaan 1, 9713 AV Groningen, The Netherlands \\ ${ }^{2}$ Laboratory of Physical Chemistry and Colloid Science, Wageningen University, Dreijenplein 6, \\ 6703 HB Wageningen, The Netherlands
}

\begin{abstract}
Using a parallel-plate flow chamber, the hydrodynamic shear forces to prevent bacterial adhesion $\left(F_{\text {prev }}\right)$ and to detach adhering bacteria $\left(F_{d e t}\right)$ were evaluated for hydrophilic glass, hydrophobic, dimethyldichlorosilane (DDS)-coated glass and six different bacterial strains, in order to test the following three hypotheses. 1. A strong hydrodynamic shear force to prevent adhesion relates to a strong hydrodynamic shear force to detach an adhering organism. 2. A weak hydrodynamic shear force to detach adhering bacteria implies that more bacteria will be stimulated to detach by passing an air-liquid interface (an air bubble) through the flow chamber. 3. DLVO (Derjaguin, Landau, Verwey, Overbeek) interactions determine the characteristic hydrodynamic shear forces to prevent adhesion and to detach adhering micro-organisms as well as the detachment induced by a passing air-liquid interface. $F_{\text {prev }}$ varied from 0.03 to $0.70 \mathrm{pN}$, while $F_{\text {det }}$ varied from 0.31 to over $19.64 \mathrm{pN}$, suggesting that after initial contact, strengthening of the bond occurs.

Generally, it was more difficult to detach bacteria from DDS-coated glass than from hydrophilic glass, which was confirmed by air bubble detachment studies. Calculated attractive forces based on the DLVO theory ( $\left.F_{D L V O}\right)$ towards the secondary interaction minimum were higher on glass than on DDS-coated glass. In general, all three hypotheses had to be rejected, showing that it is important to distinguish between forces acting parallel (hydrodynamic shear) and perpendicular (DLVO, air-liquid interface passages) to the substratum surface.
\end{abstract}

\section{INTRODUCTION}

Microbial adhesion and subsequent biofilm formation occur in many fields of industrial and medical applications, such as on ship hulls, heat exchanger plates, food packaging materials and biomaterials implants, including urinary catheters, contact lenses and vascular grafts (Costerton et al., 1999; Flemming, 2002; Von Eiff et al., 2005). Common in most applications is the deposition of micro-organisms to a surface from a flowing suspension. This implies that a variety of forces act on depositing and already adhering organisms. Deposition is mainly governed by Brownian motion, sedimentation and hydrodynamic forces, while actual adhesion of micro-organisms to a substratum surface is mediated by Lifshitz-Van der Waals, electrostatic, acid-base and hydrophobic interaction forces (Van Oss et al., 1986).

Fluid flow is an important factor in microbial deposition (Bakker et al., 2002). An increase in fluid flow velocity will

Abbreviations: AFM, atomic force microscopy; DDS, dimethyldichlorosilane; DLVO, Derjaguin, Landau, Verwey, Overbeek; PPFC, parallel-plate flow chamber. in the first instance yield increased microbial transport towards a substratum surface (convective diffusion), but at the same time cause an increase in hydrodynamic detachment forces. Shear is the dominant effect of fluid flow and can be well controlled in experimental systems, as on rotating disks, at stagnation points and in parallel-plate flow chambers. In principle, two critical shear rates can be distinguished based on current literature (see Table 1 for a summary): a critical shear rate to prevent adhesion and a critical shear rate to stimulate detachment of already adhering organisms. Both critical shear rates vary from strain to strain and also depend on the substratum material involved. The shear rates and, hence, the shear forces, required to stimulate detachment are generally higher than the shear rates to prevent adhesion.

Detachment can also be invoked by allowing an air bubble to pass over adhering bacteria. The passage of an air-liquid interface is accompanied by a perpendicularly oriented force of around $10^{-7} \mathrm{~N}$, which is much higher than the hydrodynamic shear forces acting parallel to a substratum surface. However, a passing air-liquid interface does not cause complete bacterial detachment for all combinations 
Table 1. Summary of interaction forces between bacteria and substratum surfaces, together with the method applied

Hydrodynamic forces are calculated using $F=\eta \sigma A_{p}$, in which $\eta$ is the absolute viscosity of water, $\sigma$ the shear rate and $A_{p}$ the area of the particle exposed to shear. Cocci were assumed to have a radius of $0.5 \mu \mathrm{m}$, while rod-shaped bacteria were approximated by spheres with equal volume, using $0.7 \mu \mathrm{m}$ on average as a radius. DLVO forces are taken as the attractive force towards the predicted secondary minimum in the total interaction energy curves.

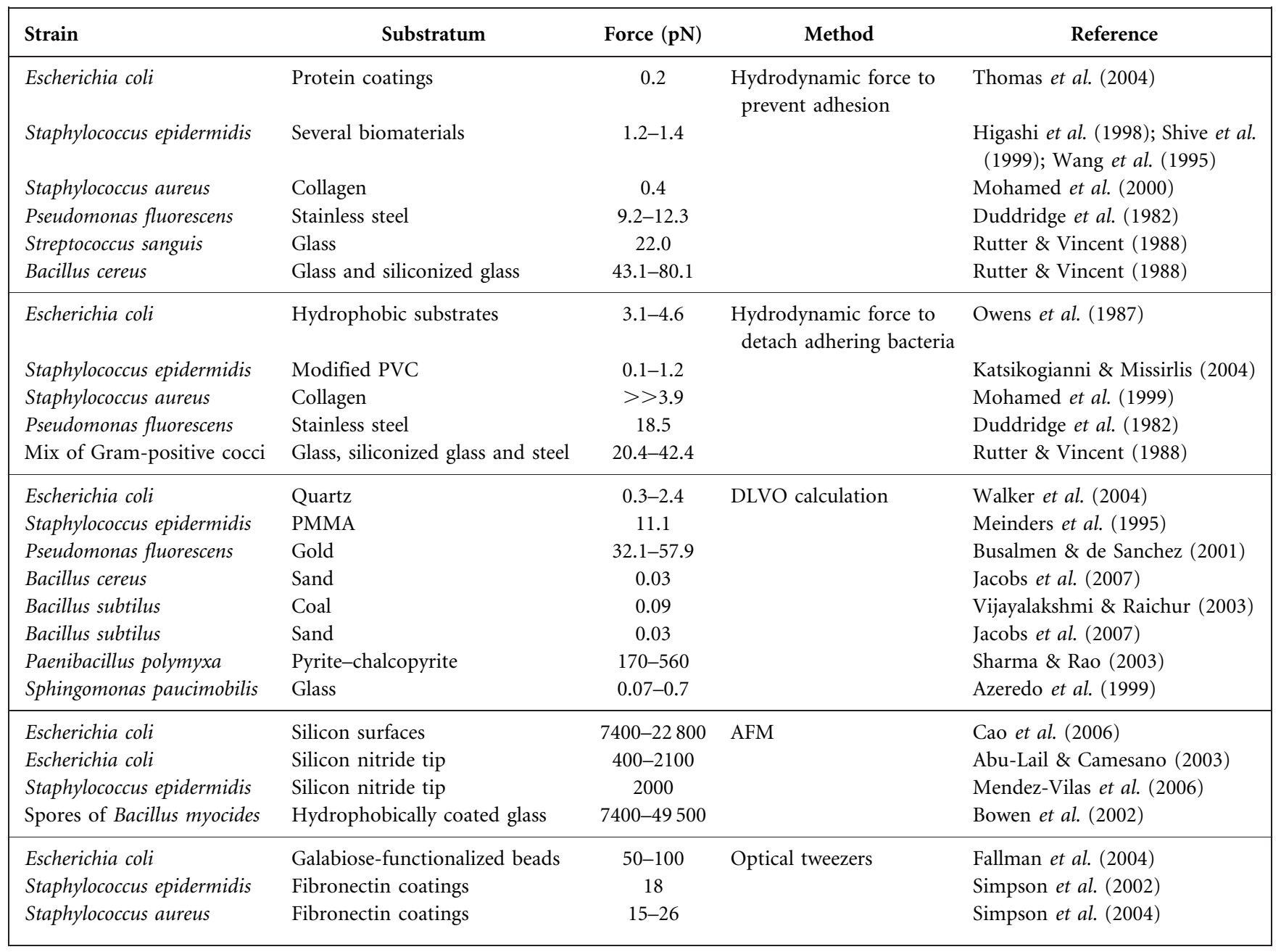

of strains and substratum surfaces. Gomez-Suarez et al. (2001) investigated detachment of several bacterial strains from hydrophilic and hydrophobic surfaces by a passing air bubble. Depending on the strain involved, the presence of a conditioning film and the velocity of the air bubble, detachment ranged from 0 to $90 \%$. Although air-bubbleinduced detachment is relatively easy to measure, it only yields an extremely rough estimate of a detachment force threshold and it cannot be used to estimate the actual binding strength.

Perpendicularly oriented interaction forces can be measured more directly, for instance using atomic force microscopy (AFM) or optical tweezers. As can be seen in Table 1, forces obtained using these techniques differ in orders of magnitude. Forces measured with optical tweezers remain in the $\mathrm{pN}$ range, while AFM yields stronger forces than any other method, which are generally in the $\mathrm{nN}$ range.
Another approach often used for assessing adhesion strength is the (extended) DLVO theory (named after Derjaguin, Landau, Verwey and Overbeek). In the DLVO theory the binding strength between colloidal particles, such as micro-organisms, and substratum surfaces may be calculated on the basis of Lifshitz-Van der Waals, (acidbase and) electrical double layer interactions. Usually, also the theoretical values provide a distinct class of force values that cannot be easily matched with experimental values, as reported in the literature.

From Table 1, it is obvious that throughout the literature different types of forces may be distinguished for every strain-substratum combination. Furthermore, conclusions on bacterial adhesion mechanisms are often based on not more than two strains (Bakker et al., 2004). Comparing all reported data is further complicated by the fact that different suspending media are used to determine adhesion 
parameters on different substrata. It is currently unclear why different methods to evaluate bacterial binding forces yield distinct classes of force values that often differ by orders of magnitude. The aim of our research is to gain more insight into the relevance of the different bacterial interaction force indicators, including theoretically predicted interaction forces from the DLVO theory, and their mutual relationships. To this end, the following hypotheses were tested.

1. A strong hydrodynamic shear force to prevent adhesion relates to a strong hydrodynamic shear force to detach an adhering organism.

2. A weak hydrodynamic shear force to detach adhering bacteria implies that more bacteria will be stimulated to detach by passing an air-liquid interface through the flow chamber.

3. DLVO interactions determine the characteristic hydrodynamic shear forces to prevent adhesion and to detach adhering micro-organisms as well as the detachment induced by a passing air-liquid interface.

To test these hypotheses, the critical shear forces to prevent bacterial adhesion and to stimulate detachment of adhering bacteria were determined. Hydrophilic glass and hydrophobic, dimethyldichlorosilane-coated glass were employed as substrata. To allow for more general conclusions to be drawn, six widely different bacterial strains were included. In addition, theoretical DLVO interaction forces, as calculated from measured zeta potentials and contact angles, were determined. Furthermore, the detachment force threshold was evaluated for detachment caused by a passing air-liquid interface.

\section{METHODS}

Bacterial strains and culture conditions. Staphylococcus epidermidis ATCC 35983, Staph. epidermidis $\mathrm{HBH}_{2}$ 169, Pseudomonas aeruginosa D1 and $P$. aeruginosa KEI 1025 were cultured aerobically from blood agar plates in $10 \mathrm{ml}$ Tryptone Soya Broth (Oxoid) for $24 \mathrm{~h}$ at $37^{\circ} \mathrm{C}$. Raoultella terrigena ATCC 33527 was precultured aerobically from nutrient agar (Nutrient Broth, Oxoid) in $10 \mathrm{ml}$ nutrient broth for $24 \mathrm{~h}$ at $37{ }^{\circ} \mathrm{C}$. Streptococcus thermophilus ATCC 19258 was precultured from a frozen stock in $10 \mathrm{ml} \mathrm{M} 17$ broth for $24 \mathrm{~h}$ at $37{ }^{\circ} \mathrm{C}$. After $24 \mathrm{~h}$, precultures were used to inoculate $200 \mathrm{ml}$ main cultures, which were grown for $16 \mathrm{~h}$ under similar conditions as the corresponding precultures. Staph. epidermidis and P. aeruginosa strains were harvested by centrifugation for $5 \mathrm{~min}$ at $5000 \mathrm{~g}, R$. terrigena and Strep. thermophilus were harvested at $10000 \mathrm{~g}$. All strains were washed twice with $10 \mathrm{mM}$ potassium phosphate buffer at $\mathrm{pH} 7$ and resuspended in the same buffer. To break bacterial chains or clusters, sonication at $30 \mathrm{~W}$ (Vibra Cell model 375, Sonics and Materials) was carried out for staphylococcal (3 times $10 \mathrm{~s}$ ) and streptococcal (2 times $10 \mathrm{~s}$ ) suspensions, while cooling in an ice/water bath. Subsequently, bacteria were resuspended to a concentration of $3 \times 10^{8}$ cells $\mathrm{ml}^{-1}$. In the calculations discussed below, the cocci were assumed to have a radius of $0.5 \mu \mathrm{m}$. Rod-shaped $P$. aeruginosa $(2.5 \mu \mathrm{m} \times 0.9 \mu \mathrm{m})$ and $R$. terrigena $(3.2 \mu \mathrm{m} \times 1.4 \mu \mathrm{m})$ were approximated as spheres with equal volume, using a radius of $0.6 \mu \mathrm{m}$ and $0.9 \mu \mathrm{m}$, respectively, as they adhere in different orientations, i.e. 'endon' and 'side-on'.
Substratum surfaces. Glass slides were sonicated for $3 \mathrm{~min}$ in $2 \%$ RBS35 (Omnilabo International) followed by thorough rinsing with tap water, demineralized water, methanol, tap water and finally demineralized water again to obtain a hydrophilic surface. After washing, the slides were either directly used or dried for $4 \mathrm{~h}$ at $80{ }^{\circ} \mathrm{C}$ prior to applying a hydrophobic coating. To obtain a hydrophobic surface, the dried glass slides were submerged for $15 \mathrm{~min}$ in a solution of dimethyldichlorosilane (DDS, Merck) in trichloroethylene $(0.05 \%, w / v)$ and washed with trichloroethylene, methanol and ultrapure water. Prepared slides were stored for no longer than 3 days at room temperature and rinsed with $10 \mathrm{mM}$ potassium phosphate buffer before use.

Bacterial adhesion in the parallel-plate flow chamber. The parallel-plate flow chamber (PPFC) and image analysis have been described previously (Busscher \& Van der Mei, 2006). The flow chamber used in this study has a length of $175 \mathrm{~mm}$, a depth of $0.75 \mathrm{~mm}$ and a width of $17 \mathrm{~mm}$. Prior to use, the flow chamber was washed with $2 \%$ Extran (Merck) and rinsed thoroughly with tap water and demineralized water before mounting a clean substratum surface in the PPFC. Subsequently, the flow chamber was installed between two communicating vessels and the system was filled with $10 \mathrm{mM}$ potassium phosphate buffer, taking care to remove all air bubbles. When the PPFC was positioned under the microscope, the vessels containing bacterial suspension were positioned at different heights to create a flow. The difference in fluid levels was maintained by a roller-pump to ensure a circulating pulse-free flow throughout the duration of an experiment. Deposition of bacteria was monitored with a phase-contrast microscope (Olympus HB-2) equipped with a $\times 40$ ultra-long-working-distance objective (Olympus ULWD-CD Plan $40 \mathrm{PL}$ ) connected to a CCD-MXRi camera (Basler A101F). Images were obtained by summation of 15 consecutive images (time interval $0.25 \mathrm{~s}$ ) in order to enhance the signal-to-noise ratio and eliminate moving bacteria from analysis. Analysis of the images was done using proprietary software based on the Matlab Image Processing Toolkit (The MathWorks).

Shear-rate dependent adhesion. The bacterial suspension was allowed to flow through the flow chamber for $1 \mathrm{~h}$ at flow rates $(Q)$ of $1,5,10,19,57,77,105$ and $153 \mathrm{ml} \mathrm{min}^{-1}$, which corresponds to shear rates $(\sigma)$ of $10,50,100,200,600,800,1100$ and $1600 \mathrm{~s}^{-1}$. Under these conditions the flow is laminar and bacterial transport occurs by convective diffusion. Adhesion was monitored on both the top (negative contribution of sedimentation) and bottom (positive contribution of sedimentation) plate of the PPFC. For each shear rate, the number of bacteria adhering per unit area was recorded as a function of time. Adhesion was then expressed in initial deposition rates $j_{0}\left(\mathrm{~cm}^{-2} \mathrm{~s}^{-1}\right)$, while at the end of each experiment an air bubble was passed through the flow chamber to stimulate detachment (only evaluated for the bottom plate).

Initial deposition rates for the top and bottom plate were averaged and expressed as deposition efficiencies by normalization with respect to the Von Smoluchowski-Levich (SL) theoretical upper limit for deposition in the PPFC. The SL upper limit for bacterial deposition is an approximate solution of the convective-diffusion equation and assumes perfect sink conditions at the substratum surface (i.e. every particle that arrives at the surface actually adheres) in the absence of sedimentation. The theoretical upper limit for deposition is given by (Elimelech, 1994):

$j_{0}^{*}=\frac{D_{\infty} c}{0.89 r}\left[\frac{2}{9} \cdot \frac{b P e}{x}\right]^{1 / 3}$

in which $D_{\infty}$ is the diffusion coefficient of the particles (taken as $3.1 \times 10^{-13} \mathrm{~m}^{2} \mathrm{~s}^{-1}$ for micron-sized bacteria: Van Holde, 1971), $c$ the 
concentration of bacteria in suspension, $r$ the bacterial radius, $x$ the longitudinal distance from the flow chamber entrance, $b$ the halfdepth of the PPFC and $P e$ the dimensionless Péclet number. This latter is defined as:

$P e=\frac{3 Q r^{3}}{4 w b^{3} D_{\infty}}$

in which $Q$ is the applied flow rate and $w$ the width of the flow chamber.

Detachment induced by a passing air-liquid interface. Following the deposition measurement, an air-liquid interface was introduced by passing an air bubble through the flow chamber, which is accompanied by a perpendicularly oriented detachment force equal to (Leenaars \& O’Brien, 1989):

$F_{\gamma}^{\max }=2 \pi \cdot r \cdot \gamma_{l v} \sin ^{2}\left(\frac{\Theta_{w, b}}{2}\right) \cos \Theta_{w, s}$ for $\Theta_{\mathrm{w}, \mathrm{s}}<90$

$F_{\gamma}^{\max }=-2 \pi \cdot r \cdot \gamma_{l v} \sin ^{2}\left(\frac{\pi+\Theta_{w, b}}{2}\right) \cos \Theta_{w, s}$ for $\Theta_{\mathrm{w}, \mathrm{s}}>90$

in which $\gamma_{l v}$ represents the interfacial surface tension of the liquid and vapour, and $\Theta_{w, b}$ and $\Theta_{w, s}$ denote the bacterial- and substratumwater contact angles, respectively.

Shear-rate-dependent detachment of adhering bacteria. The flow system was filled and positioned as described above. Bacteria were resuspended in potassium phosphate buffer to a high concentration of $7.5 \times 10^{8}$ cells $\mathrm{ml}^{-1}$ to accelerate deposition and allowed to adhere to the collector surface at a shear rate of $25 \mathrm{~s}^{-1}$. After $20 \mathrm{~min}$, flow was switched to fresh buffer without bacteria at $25 \mathrm{~s}^{-1}$ to wash out the bacterial suspension for $30 \mathrm{~min}$, after which the shear rate was increased to $250,1000,3000,6650$ or $7320 \mathrm{~s}^{-1}$ for $30 \mathrm{~min}$. The number of bacteria that remained adhering was enumerated after each step.

Surface characterization. To determine the zeta potentials of the substrata, streaming potentials were measured in $10 \mathrm{mM}$ phosphate buffer at $\mathrm{pH}$ 7. Collector surfaces were mounted in a homemade PPFC, separated by a $0.1 \mathrm{~mm}$ Teflon spacer. A platinum electrode was placed at each end of the chamber. Streaming potentials were measured at 10 different pressures ranging from $5 \times 10^{3}$ to $20 \times 10^{3}$ $\mathrm{Pa}$. Each pressure was applied for $10 \mathrm{~s}$ in both directions. Zeta potentials were deduced by linear least-squares fitting from the pressure-dependent streaming potentials (Van Wagenen \& Andrade, 1980).

For bacterial zeta potentials, bacteria were washed with demineralized water and resuspended in $10 \mathrm{mM}$ potassium phosphate buffer at $\mathrm{pH} 7$ to a concentration of $1 \times 10^{8}$ cells $\mathrm{ml}^{-1}$. The electrophoretic mobilities of these suspensions were measured at $150 \mathrm{~V}$ using a Lazer Zee Meter 501 (PenKem). The electrophoretic mobilities were converted to apparent zeta potentials assuming that the HelmholtzVon Smoluchowski approximation holds, which is appropriate considering the high value for $\kappa r$ (i.e. $\approx 150$ ) in the systems used ( $\kappa$ denotes the reciprocal Debye length, which is directly related to the ionic strength: Lyklema, 1991).

To calculate surface free energies of the substratum and bacterial cell surfaces, sessile drop contact angles were measured with water, formamide, $\alpha$-bromonaphthalene and methylene iodide. In order to measure contact angles with liquids on bacteria, bacterial lawns were prepared by depositing bacteria from suspensions in demineralized water on cellulose acetate membrane filters (Millipore, pore diameter $0.45 \mu \mathrm{m}$ ) under negative pressure until approximately 50 layers were stacked. Subsequently, filters were fixed on a sample holder and left to dry until 'plateau contact angles' could be measured, i.e. water contact angles that remained stable over time for 30-60 min. All contact angles were measured in triplicate, involving separate substrata and different bacterial cultures. Measured contact angles were converted into surface free energies using

$\cos \Theta=-1+\frac{2 \sqrt{\gamma_{s v}^{L W} \gamma_{l v}^{L W}}}{\gamma_{l v}}+\frac{2 \sqrt{\gamma_{s v}^{\text {minus }} \gamma_{l v}^{\text {minus }}}}{\gamma_{l v}}+\frac{2 \sqrt{\gamma_{s v}^{\text {plus }} \gamma_{l v}^{\text {plus }}}}{\gamma_{l v}}$

in which $\gamma_{s v}^{L W}$ is the Lifshitz-Van der Waals component of the surface free energy of the surface of interest (i.e. substratum surface or bacterial lawn) and $\gamma_{l v}$ is the surface free energy of the liquid-vapour interface. The acid-base component of the surface free energies was separated into an electron donor $\left(\gamma_{s v}^{\text {minus }}\right)$ and electron acceptor $\left(\gamma_{s v}^{\text {plus }}\right)$ parameter, according to

$\gamma_{s}^{A B}=2 \sqrt{\gamma_{s v}^{\text {minus }} \gamma_{s v}^{\text {plus }}}$

Interaction forces using the extended DLVO theory. First, the above derived surface free energy components and parameters were employed to calculate the contributions of the Lifshitz-Van der Waals $\left(\Delta G^{L W}\right)$ and acid-base $\left(\Delta G^{A B}\right)$ component to the free energy of interaction at contact in an aqueous medium between a bacterium and a substratum surface (Bos et al., 1999; Van Oss, 1994b). In the extended DLVO theory, the interaction energy is divided into a Lifshitz-Van der Waals, an acid-base and an electrostatic contribution, while accounting for their distance dependencies. The distance dependence of the Lifshitz-Van der Waals component of the interaction energies $\left(\Delta G^{L W}(d)\right)$ was calculated assuming a sphereplane geometry according to (Van Oss, 1994a):

$\Delta G^{L W}(d)=-\frac{A}{6}\left[\frac{2 r(d+r)}{d(d+2 r))}-\ln \left(\frac{d+2 r}{d}\right)\right]$

in which $d$ denotes the separation distance, which was taken as $0.157 \mathrm{~nm}$ at closest approach.

The distance dependence of the acid-base interaction energies $\left(\Delta G^{A B}(d)\right)$ were calculated according to (Van Oss, 1994a):

$\Delta G^{A B}(d)=2 \pi \cdot r \cdot \Delta G_{s l b}^{A B} \lambda \exp \left(\frac{d_{0}-d}{\lambda}\right)$

in which $\lambda$ denotes the correlation length of molecules in the liquid medium [estimated to be $0.6 \mathrm{~nm}$ (Van Oss, 1994a)] and $\Delta G_{s l b}^{A B}$ the acid-base component of the free energy of interaction at contact.

Lastly, the distance-dependent electrostatic interaction energies $\left(\Delta G^{E L}(d)\right)$ were calculated using (Norde \& Lyklema, 1989):

$\Delta G^{E L}(d)=\pi \varepsilon \varepsilon_{0} r\left(\phi_{b}^{2}+\phi_{s}^{2}\right)\left\{\frac{2 \phi_{b} \phi_{s}}{\phi_{b}^{2}+\phi_{s}^{2}} \ln \left[\frac{1+\exp (-\kappa d)}{1-\exp (-\kappa d)}\right]+\ln [1-\exp (-2 \kappa d)]\right\}(9)$

in which $\varepsilon \varepsilon_{0}$ denotes the dielectric permittivity of the medium (i.e. water), $\phi_{b}$ and $\phi_{s}$ the surface (zeta) potentials of the bacterial cell surface and collector surface, and $\kappa$ the reciprocal Debye length.

Summation and differentiation with respect to distance of these three components leads to the total DLVO interaction energy and interaction force, respectively, as a function of separation distance. All DLVO interaction forces reported in this paper represent the maximal attractive force towards the secondary interaction minimum, which was present in all bacterium-substratum systems investigated. Note that in the secondary interaction minimum, the attractive and repulsive interaction forces balance each other, and the net force equals zero. 


\section{RESULTS}

\section{Shear rates to prevent bacterial adhesion}

Fig. 1 presents an example of bacterial deposition to the bottom and top plate of the PPFC as a function of shear rate. Deposition is higher to the bottom plate than to the top plate, especially at lower shear rates. Moreover, at low shear rates an initial increase in deposition to the bottom plate can be seen with increasing shear rate up to $200 \mathrm{~s}^{-1}$ due to increased mass transport, above which deposition decreases with increasing shear due to detachment. A similar effect is observed on the top plate.

The influence of sedimentation on mass transport can be eliminated by averaging bottom and top plate depositions. Fig. 2 shows the deposition efficiencies $(\alpha)$ in the absence of sedimentation, as calculated from averaged initial deposition rates and the theoretical upper limit for deposition (equation 1) as a function of shear rate. From Fig. 2, critical shear rates to prevent adhesion $\left(\sigma_{\text {prev }}\right)$ were deduced using

$\alpha=\alpha_{0} \cdot \exp \left\{-\frac{\sigma}{\sigma_{\text {prev }}}\right\}$

where $\alpha_{0}$ is the extrapolated deposition efficiency in the absence of shear. Subsequently, values for $\sigma_{\text {prev }}$ were expressed in shear forces using

$F_{i}=A_{p} \cdot \eta \cdot \sigma_{i}$

in which $\eta$ is the absolute viscosity of the buffer $\left(1 \times 10^{-3}\right.$ Pa s) and $A_{p}$ is the area of the adhering bacterium subject to shear flow. The subscript ' $i$ ' denotes the type of hydrodynamic force calculated: prev for the hydrodynamic force to prevent adhesion and det for the hydrodynamic force to detach adhering micro-organisms. Hydrodynamic shear forces to prevent adhesion $\left(F_{\text {prev }}\right)$ are listed in Table 2. All values for $F_{\text {prev }}$ remain in the low $\mathrm{pN}$ range and are influenced by the substratum surface, although they are not consistently higher on either of the two surfaces.

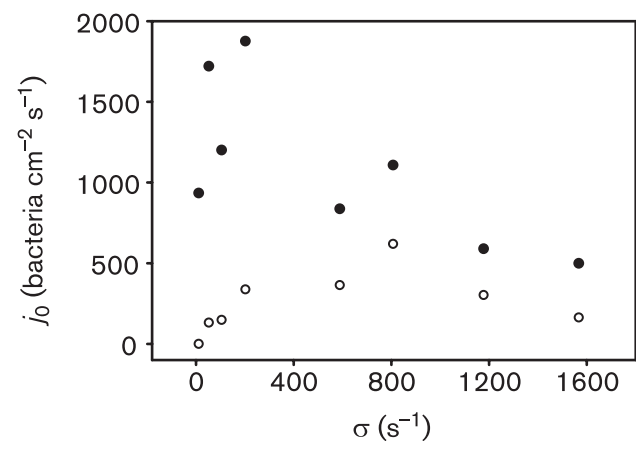

Fig. 1. Initial deposition rates (jo) for Staph. epidermidis ATCC 35983 on the bottom $(\bullet)$ and top $(\bigcirc)$ plate in a PPFC as a function of the shear rate $(\sigma)$ applied on glass.
Depending on the strain used, the difference between $F_{\text {prev }}$ on glass and DDS-coated glass can be as large as a factor of 6 .

\section{Shear rates to remove adhering bacteria}

Fig. 3 presents the detachment of bacteria from glass and DDS-coated glass as a function of the shear rate applied, expressed as the fraction $(f)$ of bacteria removed from the substratum surface. For a given shear rate, $f$ is defined as the number of removed bacteria after $30 \mathrm{~min}$ exposure to that shear divided by the number of adhering bacteria before application of the shear. From the plots in Fig. 3 critical shear rates to detach adhering bacteria $\left(\sigma_{\text {det }}\right)$ were derived, defined as the shear rate at which $63 \%$ of the adhering bacteria had detached. Subsequently, these shear rates were expressed in detachment forces $\left(F_{\text {det }}\right)$ using equation 11, and their values are listed in Table 2 . In most cases, bacteria were more readily detached from glass than from DDS-coated glass. All forces remain in the $\mathrm{pN}$ range, but are an order of magnitude larger than $F_{\text {prev }}$. Note that the critical detachment level could not be reached within the range of shear rates possible in our experimental set-up for Staph. epidermidis ATCC 35983 on DDS and for R. terrigena ATCC 33527 on glass.

\section{Air-bubble-induced bacterial detachment}

Table 3 summarizes the effect of an air bubble passing over the adhering bacteria. At first sight, binding affinity on DDS-coated glass seems to be less than on hydrophilic glass, as judged from air-bubble-induced detachment. However, on DDS-coated glass, the force exerted by an air-liquid interface on adhering bacteria is calculated to be up to five times larger than on glass. For the two Staph. epidermidis strains and $R$. terrigena, this results in higher detachment percentages from DDS-coated glass. In contrast, for the pseudomonas strains and Strep. thermophilus the percentages detached from glass and from DDS-coated glass are not significantly different. It should be noted that detachment by a passing air bubble does not give any indication of the magnitude of the interaction forces. For example, for the staphylococcal strains and $R$. terrigena on glass, it cannot be established at what force detachment would be stimulated to a larger extent. Air bubble detachment studies are inconclusive here with respect to binding strength information. However, for the pseudomonas strains and Strep. thermophilus it is clear that, even though the exerted force on DDS-coated glass is stronger, detachment percentages are not higher. Results for these strains suggest stronger interaction forces with the hydrophobic DDS-coated glass.

\section{Surface characterization and calculation of theoretical interaction forces}

Measured contact angles, together with the surface free energy components of the wetting liquids used, are listed in 


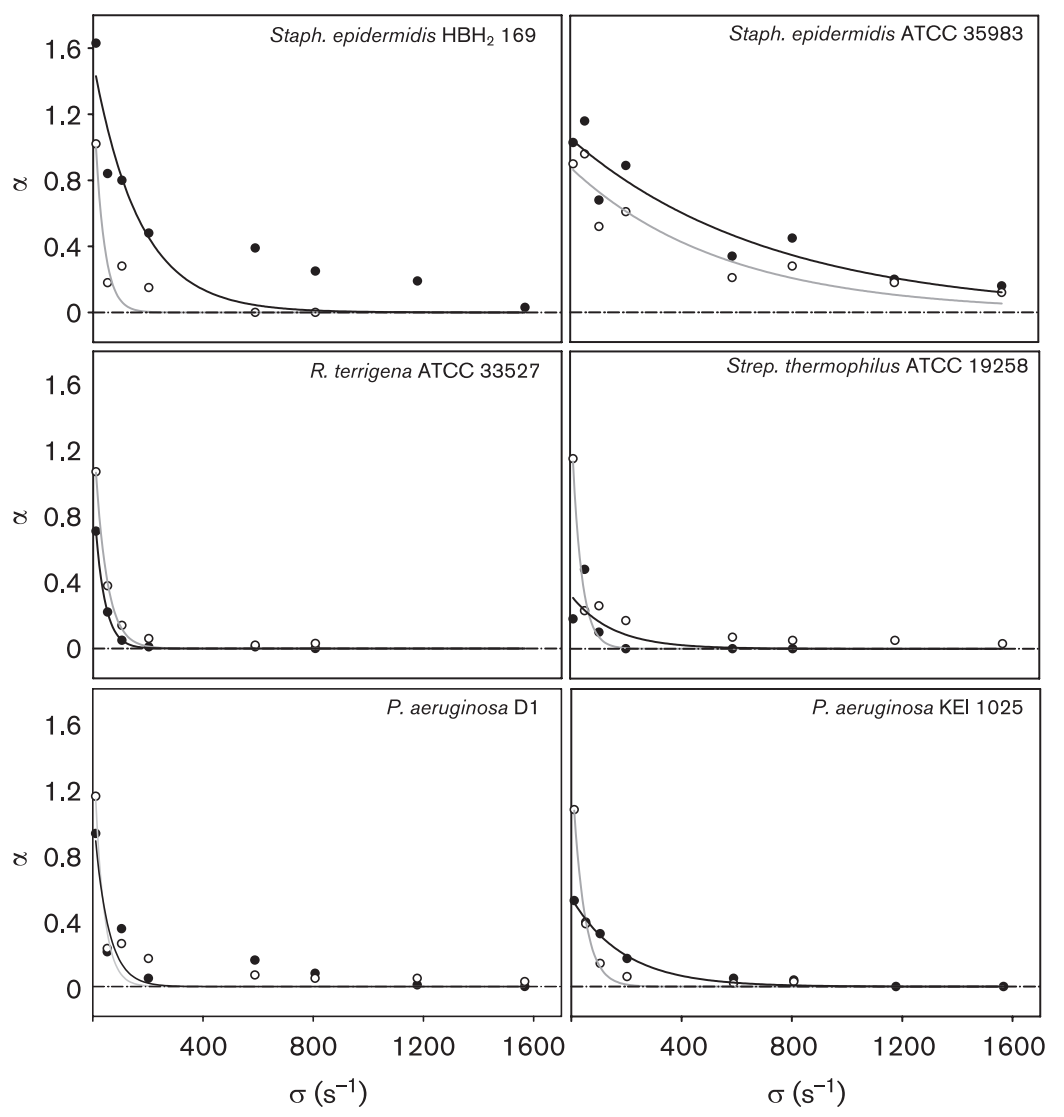

Fig. 2. Bacterial deposition efficiency $(\alpha)$ in the absence of a mass-transport contribution from sedimentation as a function of the shear rate $(\sigma)$ applied on glass $(\bullet)$ and DDS-coated glass $(\bigcirc)$ for the six bacterial strains studied. Black and grey lines represent the fits of equation 10 to the data points on glass and DDS-coated glass, respectively.

Table 4. All bacteria have a surface hydrophilicity comparable to that of glass, as judged from the water contact angles. DDS-coated glass is significantly more hydrophobic. Bacterial cell surfaces and the glass substratum surface are predominantly electron-donating, as evidenced by their larger $\gamma^{\text {minus }}$ surface free energy parameter as compared with $\gamma^{\text {plus }}$. Hydrophobic, DDScoated glass is neither a good electron donor nor acceptor. All surfaces are negatively charged and whereas bacterial zeta potentials vary between -22 and $-50 \mathrm{mV}$, the zeta potentials of glass and DDS-coated glass are similarly negative $(-33$ to $-35 \mathrm{mV})$.

The bacterial cell and substratum surface properties listed in Table 4 have been used in the extended DLVO theory, yielding interaction free energy- and force-distance profiles for all combinations of bacteria and substratum surfaces, as illustrated in Fig. 4 for P. aeruginosa KEI 1025. Note the reversed force-axis (right-hand side) in Fig. 4, indicating that negative values correspond to attractive

Table 2. Critical shear forces to prevent $\left(F_{\text {prev }}\right)$ bacterial adhesion and to detach $\left(F_{\text {det }}\right)$ adhering bacteria from a hydrophilic (glass) and hydrophobic (DDS-coated) substratum, together with the theoretically calculated DLVO interaction forces

Reported uncertainties are based on the standard error of the predicted fitting curve.

\begin{tabular}{|c|c|c|c|c|c|c|}
\hline \multirow[t]{2}{*}{ Bacterial strain } & \multicolumn{2}{|c|}{$F_{\text {prev }}(\mathrm{pN})$} & \multicolumn{2}{|c|}{$F_{d e t}(\mathrm{pN})$} & \multicolumn{2}{|c|}{$F_{D L V O}(\mathrm{pN})$} \\
\hline & Glass & DDS & Glass & DDS & Glass & DDS \\
\hline Staph. epidermidis ATCC 35983 & $0.57 \pm 0.22$ & $0.10 \pm 0.03$ & $5.39 \pm 0.19$ & $>5.75^{\star}$ & 0.05 & 0.03 \\
\hline R. terrigena ATCC 33527 & $0.10 \pm 0.00$ & $0.11 \pm 0.01$ & $>19.64^{*}$ & $14.30 \pm 2.45$ & 0.10 & 0.06 \\
\hline Strep. thermophilus ATCC 19258 & $0.12 \pm 0.34$ & $0.03 \pm 0.01$ & $0.55 \pm 0.05$ & $0.68 \pm 0.04$ & 0.00 & 0.00 \\
\hline
\end{tabular}

${ }^{\star}$ No detachment could be stimulated within the shear rates applied, and the value indicated denotes the highest shear applied. 


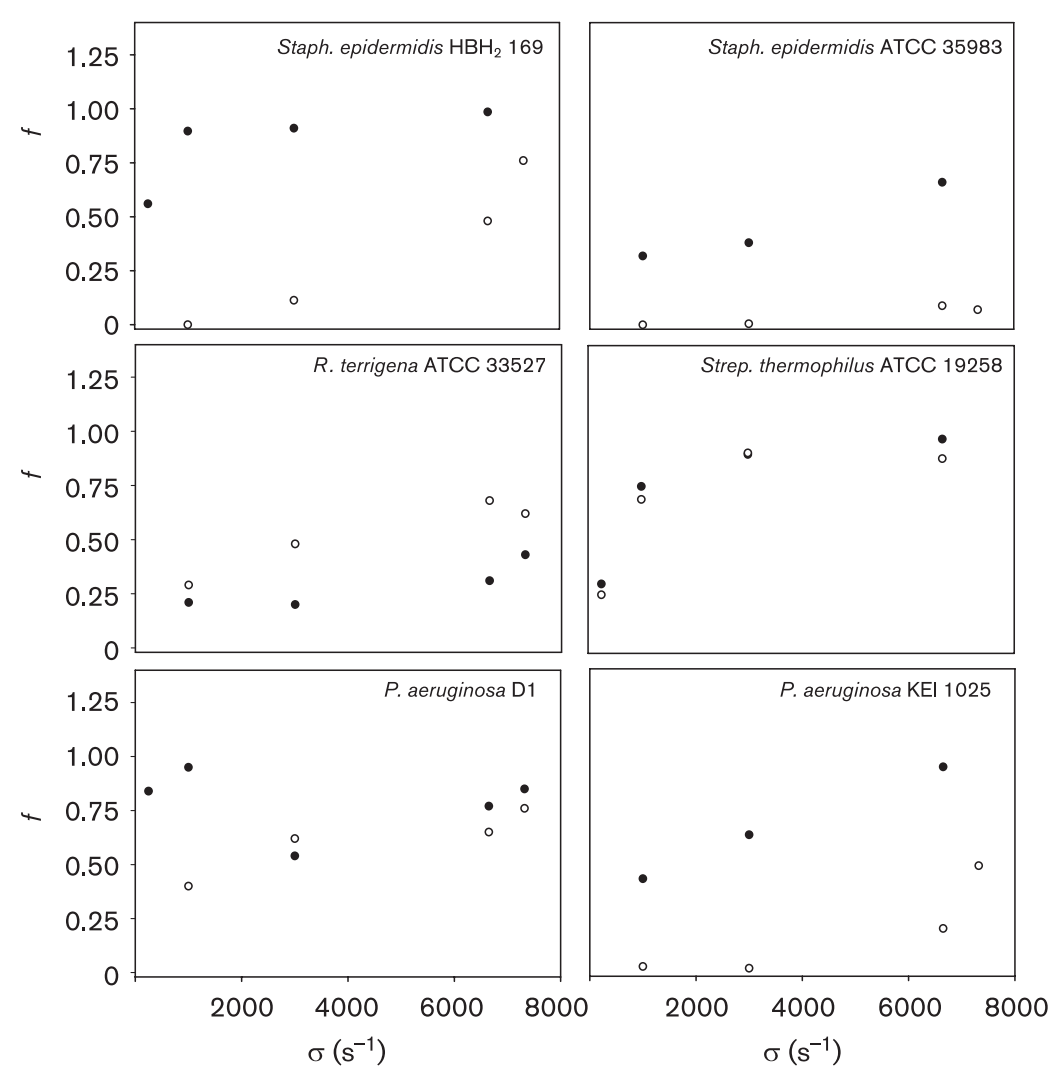

Fig. 3. Shear-induced detachment, expressed as the fraction $(f)$ of bacteria that are removed, as a function of the shear rate $(\sigma)$ applied for glass ( $)$ and DDS-coated glass $(\bigcirc)$ after $30 \mathrm{~min}$ of flow. interaction forces according to the definition of force:

$F(d)=-\frac{\delta}{\delta d} E(d)$

Residing in the secondary minimum of the interaction energy corresponds to zero interaction force, resulting from compensating attractive (Van der Waals) and repulsive (electrostatic) forces. However, the approach towards the secondary minimum yields a maximum net attraction force (Fig. 4) at a distance of about $40 \mathrm{~nm}$ from the surface. On glass, these interaction forces are generally higher than on DDS-coated glass (see also Table 2), due to larger Hamaker constants for glass as a substratum. Additionally, on DDS-coated glass a primary minimum (closer to the surface) is predicted due to acid-base interaction. The height of the energy barrier between the secondary and primary minimum varies from $229 k T$ for Strep. thermophilus to $1030 \mathrm{kT}$ for $R$. terrigena and therefore it is very unlikely that a depositing microorganism will cross the barrier to adhere in the primary minimum. On glass, a primary interaction minimum is absent.

Table 3. Air-bubble-induced detachment

The table shows the number of adhering bacteria on the bottom plate of the PPFC after $1 \mathrm{~h}$ of flow ( $N_{1 h}$, averaged over adhesion experiments at $\sigma=10,50,100$ and $200 \mathrm{~s}^{-1} ; n=1$ for each shear rate), detachment percentages from glass and a DDS-coating and the corresponding maximal detachment force $\left(F_{\max }\right)$ that a liquid/air interface exerts.

\begin{tabular}{|c|c|c|c|c|c|c|}
\hline \multirow[t]{2}{*}{ Strain } & \multicolumn{3}{|c|}{ Glass } & \multicolumn{3}{|c|}{ DDS } \\
\hline & $10^{-6} \times N_{l h} \mathrm{~cm}^{-2}$ & Detachment (\%) & $F_{\max }(\mathrm{nN})$ & $10^{-6} \times N_{l h} \mathrm{~cm}^{-2}$ & Detachment (\%) & $F_{\max }(\mathrm{nN})$ \\
\hline Staph. epidermidis $\mathrm{HBH}_{2} 169$ & $4.9 \pm 0.5$ & $9 \pm 10$ & 14 & $3.8 \pm 0.5$ & $92 \pm 9$ & 40 \\
\hline Staph. epidermidis ATCC 35983 & $4.0 \pm 0.8$ & $4 \pm 5$ & 20 & $3.5 \pm 0.8$ & $62 \pm 47$ & 39 \\
\hline R. terrigena ATCC 33527 & $0.8 \pm 0.8$ & $27 \pm 6$ & 16 & $1.4 \pm 1.0$ & $87 \pm 14$ & 72 \\
\hline Strep. thermophilus ATCC 19258 & $0.4 \pm 0.3$ & $56 \pm 16$ & 17 & $0.5 \pm 0.5$ & $47 \pm 21$ & 39 \\
\hline$P$. aeruginosa $\mathrm{D} 1$ & $0.3 \pm 0.4$ & $71 \pm 40$ & 37 & $0.4 \pm 0.2$ & $40 \pm 14$ & 48 \\
\hline P. aeruginosa KEI 1025 & $1.3 \pm 2.1$ & $53 \pm 10$ & 12 & $2.9 \pm 0.7$ & $51 \pm 13$ & 54 \\
\hline
\end{tabular}


Table 4. Physico-chemical characteristics of the bacterial strains and collector surfaces used

Bacterial characterizations were based on three separately grown cultures. Per culture, contact angles of water $\left(\Theta_{w}\right)$, formamide $\left(\Theta_{\text {form }}\right), \alpha-$ bromonaphthalene $\left(\Theta_{b r}\right)$ and methylene iodide $\left(\Theta_{\text {met }}\right)$ were measured on four bacterial lawns using one droplet per liquid per bacterial lawn. Zeta potentials $(\zeta)$ were determined in triplicate. Contact angle and streaming potential measurements on substratum surfaces were performed in quadruplicate. Free surface energy components are derived from contact angle measurements giving an electron-donating $\left(\gamma^{\text {minus }}\right)$ and -accepting $\left(\gamma^{p l u s}\right)$ parameter for the acid-base component $\left(\gamma^{A B}\right)$, the Lifshitz-Van der Waals component $\left(\gamma^{L W}\right)$ and the total surface free energy $\left(\gamma^{T o t}\right)$.

\begin{tabular}{|c|c|c|c|c|c|c|c|c|c|c|}
\hline Bacterial strain & $\Theta_{w}\left({ }^{\circ}\right)$ & $\Theta_{\text {form }}\left({ }^{\circ}\right)$ & $\Theta_{b r}\left({ }^{\circ}\right)$ & $\Theta_{m e t}\left({ }^{\circ}\right)$ & $\begin{array}{c}\gamma^{\text {minus }} \\
\left(\mathrm{mJ} \mathrm{\mathbf {m } ^ { - 2 }}\right)\end{array}$ & $\begin{array}{c}\gamma^{\text {plus }} \\
\left(\mathrm{mJ} \mathbf{m}^{-2}\right)\end{array}$ & $\begin{array}{c}\gamma^{A B} \\
\left(\mathrm{~mJ} \mathrm{~m}^{-2}\right)\end{array}$ & $\begin{array}{c}\gamma^{L W} \\
\left(\mathrm{~mJ} \mathbf{m}^{-2}\right)\end{array}$ & $\begin{array}{c}\gamma^{T o t} \\
\left(\mathrm{~m} \mathbf{J} \mathbf{m}^{-2}\right)\end{array}$ & $\zeta(\mathrm{mV})$ \\
\hline Staph. epidermidis $\mathrm{HBH}_{2} 169$ & $31 \pm 4$ & $31 \pm 4$ & $34 \pm 5$ & $50 \pm 3$ & 47.8 & 0.4 & 9 & 40 & 49 & $-50 \pm 6$ \\
\hline R. terrigena ATCC 33527 & $24 \pm 3$ & $24 \pm 3$ & $40 \pm 4$ & $51 \pm 4$ & 49.9 & 1.8 & 19 & 34 & 53 & $-49 \pm 5$ \\
\hline Strep. thermophilus ATCC 19258 & $35 \pm 2$ & $31 \pm 4$ & $58 \pm 2$ & $77 \pm 2$ & 41.2 & 5.3 & 30 & 22 & 52 & $-22 \pm 5$ \\
\hline P. aeruginosa $\mathrm{D} 1$ & $44 \pm 6$ & $42 \pm 4$ & $48 \pm 8$ & $58 \pm 6$ & 38.8 & 1.2 & 14 & 30 & 44 & $-30 \pm 3$ \\
\hline \multicolumn{11}{|l|}{ Substratum surface } \\
\hline Glass & $28 \pm 8$ & $25 \pm 3$ & $51 \pm 2$ & $64 \pm 1$ & 45.8 & 3.7 & 26 & 28 & 54 & $-35 \pm 5$ \\
\hline DDS-coated glass & $101 \pm 2$ & $85 \pm 3$ & $59 \pm 4$ & $65 \pm 4$ & 2.2 & 0.0 & 0 & 26 & 26 & $-33 \pm 2$ \\
\hline
\end{tabular}

\section{DISCUSSION}

The forces that govern microbial deposition, adhesion and detachment are still not fully understood, and are difficult to relate to each other. In a previous study we investigated the characteristic shear force to prevent adhesion of microbial strains (Roosjen et al., 2005). In the current research we used a more systematic approach by including not only the shear forces to prevent adhesion, but also those that stimulate detachment of adhering bacteria, as

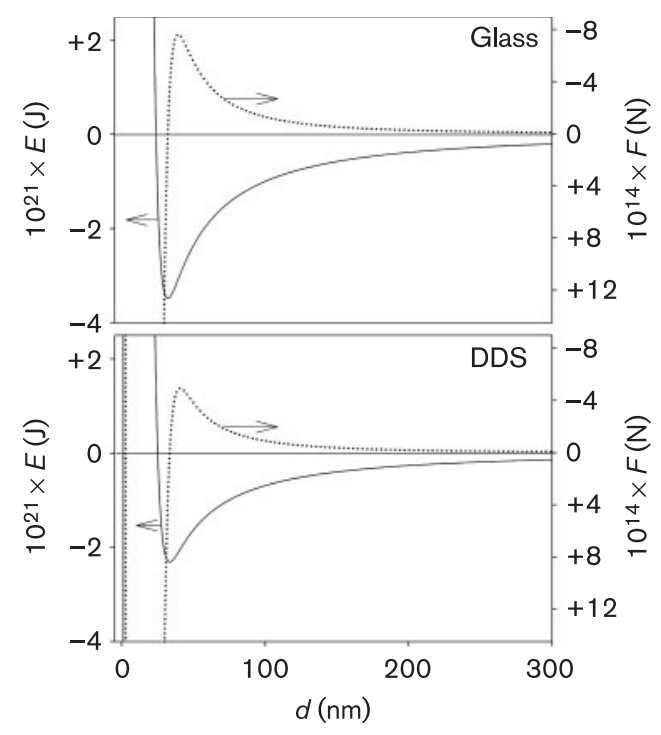

Fig. 4. Example of the extended DLVO interaction energy (full lines) and force (dotted lines) as a function of distance for $P$. aeruginosa KEI 1025 on glass and DDS-coated glass. Arrows indicate the correct axis for the two plots. Note the reversed force-axis. well as theoretical adhesion forces calculated using the extended DLVO theory. In addition, the effect of a passing air-liquid interface, which subjects adhering bacteria to a high, perpendicularly oriented detachment force, was determined. Furthermore, all experiments were carried out with six different bacterial strains in order to allow general conclusions to be drawn. As a first step in the experimental analysis, the gravitational force and its impact on bacterial deposition (Agladze et al., 2005; Walt et al., 1985) and adhesion was eliminated by averaging the deposition rates on the bottom and top plate. At low shear rates, deposition efficiencies $(\alpha)$ exceed unity, especially for the Staph. epidermidis strains, indicating that deposition is more favourable than theoretically predicted. Often such deviations are ascribed to the presence of surface structures (Triandafillu et al., 2003).

With respect to possible relations between the different forces distinguished, we have tested the following hypotheses:

(1) A strong hydrodynamic shear force to prevent adhesion relates to a strong hydrodynamic shear force to detach an adhering organism. This hypothesis implies a positive correlation between attachment and detachment. Comparison between $F_{\text {prev }}$ and $F_{\text {det }}$ (Table 2) shows that regardless of the substratum involved, $F_{d e t}$ is always larger than $F_{\text {prev }}$. In the experimental set-up used, bacteria had adhered to the substratum surface for at least half an hour before being subject to high shear. Over time, the bond between a bacterium and the substratum surface may become stronger. Supporting evidence for this is provided by others who have used AFM and found that the adhesion force increases with prolonged contact time (VadilloRodriguez et al., 2004; Xu et al., 2005). Thus, even though initial adhesion forces are rather weak, they may be indicative of forces after a prolonged time, i.e. a relatively strong $F_{\text {prev }}$ might be expected to correspond to a relatively 
strong $F_{\text {det }}$. However, from Fig. 5 it is clear that no correlation exists between $F_{\text {prev }}$ and $F_{\text {det }}$. This implies that attachment and detachment should be regarded as independent processes and the hypothesis of an unambiguous relation between attachment and detachment forces should be rejected.

(2) A weak hydrodynamic shear force to detach adhering bacteria implies that more bacteria will be stimulated to detach by passing an air-liquid interface through the flow chamber. Table 2 clearly indicates that $F_{\text {det }}$ for hydrophobic DDS-coated glass is larger than $F_{\text {det }}$ for hydrophilic glass, indicating stronger interaction forces on the hydrophobic substratum. Table 3 summarizes parameters involved in air-bubble-induced detachment. An air-liquid interface exerts forces $10^{4}$ times larger than $F_{\text {det }}$, yet it does not result in complete detachment. Combining the data in Tables 2 and 3 reveals the absence of a clear relation between shearinduced detachment and detachment by passing an air bubble. Thus a weaker $F_{\text {det }}$ does not result in higher airbubble-stimulated detachment and this hypothesis has to be rejected too. In this respect it must be realized that different mechanisms of detachment are involved in the two processes. Hydrodynamic detachment forces are measured while the system is completely submerged in liquid whereas an extra phase is introduced in air-bubbleinduced detachment. Furthermore, $F_{d e t}$ is a force acting parallel to the substratum surface, whereas the air-liquid interface acts perpendicularly to the substratum surface.

(3) DLVO interactions determine the characteristic hydrodynamic shear forces to prevent adhesion and to detach adhering micro-organisms as well as the detachment induced by a passing air-liquid interface. Further analysis revealed the absence of quantitative relations between $F_{D L V O}$ and $F_{\text {prev }}$ as well as between $F_{D L V O}$ and $F_{d e t}$ (Fig. 5). DLVO predictions have often been demonstrated to deviate from experimental observations of bacterial interaction phenomena, which is usually ascribed to the presence of surface appendages (Jucker et al., 1998; Ong et al., 1999) or chemical surface heterogeneities. However, the direction of action of the DLVO forces should be taken into account as well. DLVO forces act perpendicularly to the substratum surface, whereas both $F_{\text {prev }}$ and $F_{\text {det }}$ are directed parallel to the substratum surface.

When the fluid flow is increased to high enough values, the bacterium most likely detaches in a rolling fashion (Das et al., 1994). It can be argued that in this mode of detachment, forces normal to the surface (i.e. DLVO and lift forces) are related to forces directed parallel to the surface. However, in similar detachment studies it was found that lift forces are negligible and surface roughness may play a decisive role in determining the hydrodynamic force to remove adhering particles from the surface (Batra et al., 2001; Yiantsios \& Karabelas, 1995). This feature is not accounted for in the DLVO theory. Table 2 shows only slight differences between the theoretical $F_{D L V O}$ values for the various microbial strains, but substantial differences between the experimentally obtained forces $F_{\text {prev }}$ and $F_{\text {det }}$. If a correlation between DLVO forces and shear forces did exist, an increase of these parallel-directed forces would imply an increase in normally directed forces. However, this is not observed in $F_{D L V O}$. Hence, the parallel detachment forces do not correlate with the perpendicularly directed DLVO forces.

The DLVO theory predicts a secondary minimum of interaction at a distance of about $30-40 \mathrm{~nm}$ from the surface (see Fig. 4). On hydrophilic glass, closer approach is impossible due to strong repulsion, and adhesion can only occur in the secondary minimum. On DDS-coated glass,
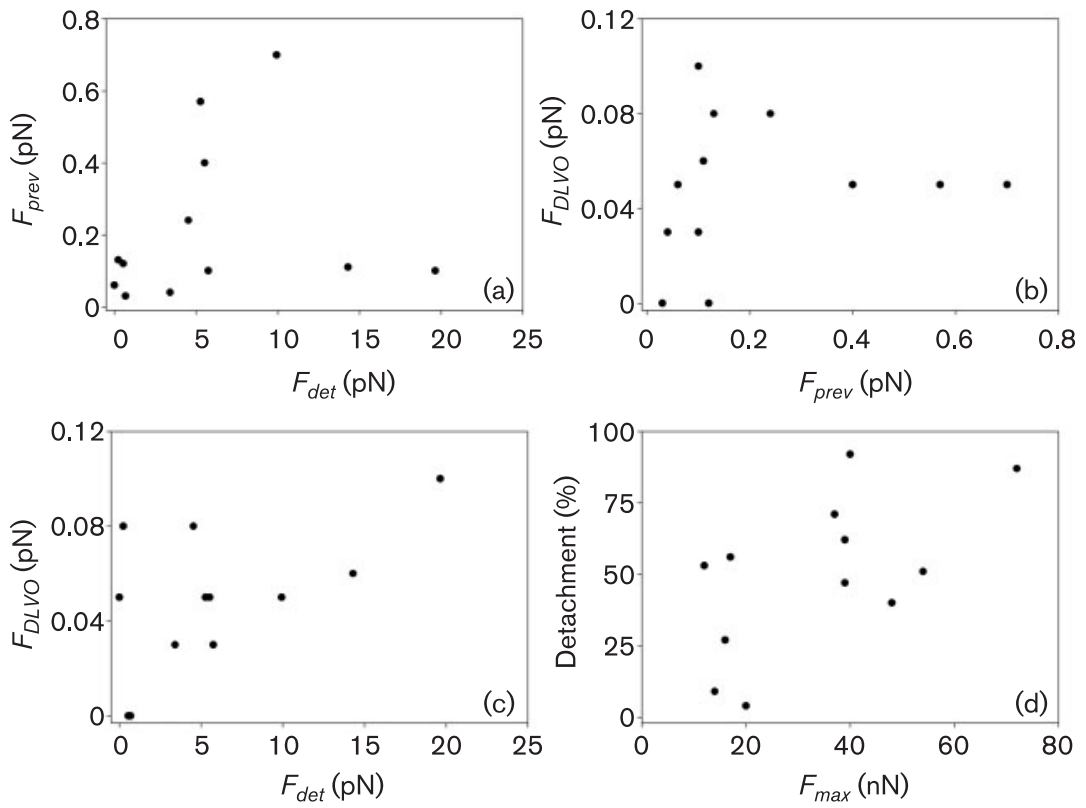

Fig. 5. Graphical representation of possible relations between $F_{p r e v}$ and $F_{d e t}(\mathrm{a}), F_{D L V O}$ and $F_{\text {prev }}(\mathrm{b}), F_{D L v O}$ and $F_{\text {det }}(\mathrm{c})$, and detachment percentage and $F_{\text {max }}(d)$. 
also primary minimum interactions are predicted. However, due to the prohibitively high barrier of the free energy (ranging from 229 to $1030 k T$ depending on the strain used), it is very unlikely that adhesion in the primary minimum can occur. Therefore, also on the hydrophobic, DDS-coated glass, only adhesion in the secondary minimum is expected to occur. As can be seen in Table 2, $F_{\text {det }}$ values are much higher than $F_{D L V O}$. Often, a transition of adhesion from the secondary interaction minimum towards the primary minimum is used as explanation (Van Loosdrecht \& Zehnder, 1990). However, in this study this is considered to be impossible, as on glass a primary minimum is absent and on DDS-coated glass it is considered to be unreachable due to the high energy barrier. It is therefore more likely that the higher $F_{\text {det }}$ values are the result of attachment of surface appendages, or 'extracellular polymeric substances' produced, capable of reaching the surface. These structures are known to extend as much as hundreds of nanometres away from the bacterial cell wall (Bos et al., 1999), which is more than enough to bridge the distance between the secondary minimum and the substratum surface. Unfortunately, although it is known for instance that some streptococci may possess surface fibrils, structural information about the cell surface of most of the strains studied in the literature is lacking, let alone detailed knowledge about the length, diameter and micro(nano-)scopic physico-chemical properties of these structures. The use of the DLVO theory as currently done in the literature, as well as in this paper, can therefore only pertain to long-distance approach, where fine surface structures do not play a role. Up to what distance of approach and to what extent this statement is valid, is hard to say. However, while the DLVO theory predicts interactions for the entire micro-organism, it is likely that the experimentally obtained detachment forces are related to a number of distinct (hydrogen) bonds. When these linkages break, due to parallel-directed forces, the bacterium can be transported away from the surface due to lift forces which are induced by the tangential flow (Cantat \& Misbah, 1999). In this respect, parallel-directed hydrodynamic forces (i.e. $F_{\text {prev }}$ and $F_{d e t}$ ) can serve as useful parameters to indicate adhesion strength.

When combining the detachment parameters (i.e. $F_{d e t}$ and the air bubble detachment percentage), our results suggest that bridging between the bacterium and the substrate surface is more favourable for DDS-coated glass. $F_{d e t}$ on hydrophobic DDS-coated glass is always higher than on glass and even though one has to be cautious in interpreting air bubble detachment percentages, the higher detachment force exerted by the air bubble on DDS-coated glass does not necessarily lead to more detachment. The hydrophobicity of the surface probably enhances the possibility of bridging, as removal of water from between the interacting surfaces is more favourable. This matter is further complicated by the influence of the type of medium in which adhesion occurs. The DLVO theory is based on averaged properties of the surfaces of the bacterial cell and substratum. However, it was found that ions in the suspending medium, especially divalent ions, can greatly influence the adhesion of bacteria to a surface, probably due to surface charge heterogeneities resulting from complexation of different ions with the (bacterial cell) surface(s) (De Kerchove \& Elimelech, 2008). Since our experiments were performed in potassium phosphate buffer, we cannot rule out similar effects caused by the divalent phosphate anion.

Even though no quantitative correlation between the DLVO theory and detachment behaviour could be established, and hypothesis (3) should therefore be rejected, this theory does help to provide a better insight into the mechanism of bacterial adhesion to a substratum surface.

\section{Conclusions}

The hydrodynamic force to prevent adhesion is lower than the hydrodynamic force to stimulate detachment, showing that the bond between a substratum surface and a bacterium becomes stronger after initial adhesion. Consequently, $F_{\text {prev }}$ and $F_{\text {det }}$ should be considered as independent parameters.

There is no unambiguous relation between the hydrodynamic forces $\left(F_{\text {prev }}\right.$ and $\left.F_{\text {det }}\right)$ directed parallel to the substratum surface and perpendicularly oriented parameters $\left(F_{D L V O}\right.$, air-liquid interface detachment), because these forces act in different directions. DLVO forces may be incorrectly estimated because of local charge heterogeneities and bridging between cell appendages and/or exudates on the one hand and substrate surface on the other. Furthermore, air-liquid-interface-induced detachment relies on a three-phase system, whereas the other forces are obtained for a two-phase environment, complicating establishment of a possible correlation.

\section{REFERENCES}

Abu-Lail, N. I. \& Camesano, T. A. (2003). Role of lipopolysaccharides in the adhesion, retention, and transport of Escherichia coli JM109. Environ Sci Technol 37, 2173-2183.

Agladze, K., Wang, X. \& Romeo, T. (2005). Spatial periodicity of Escherichia coli K-12 biofilm microstructure initiates during a reversible, polar attachment phase of development and requires the polysaccharide adhesin PGA. J Bacteriol 187, 8237-8246.

Azeredo, J., Visser, J. \& Oliveira, R. (1999). Exopolymers in bacterial adhesion: interpretation in terms of DLVO and XDLVO theories. Colloids Surf B Biointerfaces 14, 141-148.

Bakker, D. P., Busscher, H. J. \& Van der Mei, H. C. (2002). Bacterial deposition in a parallel plate and a stagnation point flow chamber: microbial adhesion mechanisms depend on the mass transport conditions. Microbiology 148, 597-603.

Bakker, D. P., Postmus, B. R., Busscher, H. J. \& Van der Mei, H. C. (2004). Bacterial strains isolated from different niches can exhibit different patterns of adhesion to substrata. Appl Environ Microbiol 70, 3758-3760. 
Batra, A., Paria, S., Manohar, C. \& Khilar, K. C. (2001). Removal of surface adhered particles by surfactants and fluid motions. AIChE J 47, 2557-2565.

Bos, R., Van der Mei, H. C. \& Busscher, H. J. (1999). Physicochemistry of initial microbial adhesive interactions - its mechanisms and methods for study. FEMS Microbiol Rev 23, 179-230.

Bowen, W. R., Fenton, A. S., Lovitt, R. W. \& Wright, C. J. (2002). The measurement of Bacillus mycoides spore adhesion using atomic force microscopy, simple counting methods, and a spinning disk technique. Biotechnol Bioeng 79, 170-179.

Busalmen, J. P. \& de Sanchez, S. R. (2001). Adhesion of Pseudomonas fluorescens (ATCC 17552) to nonpolarized and polarized thin films of gold. Appl Environ Microbiol 67, 3188-3194.

Busscher, H. J. \& Van der Mei, H. C. (2006). Microbial adhesion in flow displacement systems. Clin Microbiol Rev 19, 127-141.

Cantat, I. \& Misbah, C. (1999). Lift force and dynamical unbinding of adhering vesicles under shear flow. Phys Rev Lett 83, 880-883.

Cao, T., Tang, H. Y., Liang, X. M., Wang, A. F., Auner, G. W., Salley, S. O. \& Ng, K. Y. S. (2006). Nanoscale investigation on adhesion of $E$. coli surface modified silicone using atomic force microscopy. Biotechnol Bioeng 94, 167-176.

Costerton, J. W., Stewart, P. S. \& Greenberg, E. P. (1999). Bacterial biofilms: a common cause of persistent infections. Science 284, 13181322.

Das, S. K., Schechter, R. S. \& Sharma, M. M. (1994). The role of surface-roughness and contact deformation on the hydrodynamic detachment of particles from surfaces. J Colloid Interface Sci 164, 63-77.

De Kerchove, A. J. \& Elimelech, M. (2008). Calcium and magnesium cations enhance the adhesion of motile and nonmotile Pseudomonas aeruginosa on alginate films. Langmuir 24, 3392-3399.

Duddridge, J. E., Kent, C. A. \& Laws, J. F. (1982). Effect of surface shear-stress on the attachment of Pseudomonas fluorescens to stainlesssteel under defined flow conditions. Biotechnol Bioeng 24, 153-164.

Elimelech, M. (1994). Particle deposition on ideal collectors from dilute flowing suspensions - mathematical formulation, numericalsolution, and simulations. Sea Technol 4, 186-212.

Fallman, E., Schedin, S., Jass, J., Andersson, M., Uhlin, B. E. \& Axner, O. (2004). Optical tweezers based force measurement system for quantitating binding interactions: system design and application for the study of bacterial adhesion. Biosens Bioelectron 19, 1429-1437.

Flemming, H. C. (2002). Biofouling in water systems - cases, causes and counter measures. Appl Microbiol Biotechnol 59, 629-640.

Gomez-Suarez, C., Busscher, H. J. \& Van der Mei, H. C. (2001). Analysis of bacterial detachment from substratum surfaces by the passage of air-liquid interfaces. Appl Environ Microbiol 67, 25312537.

Higashi, J. M., Wang, I. W., Shlaes, D. M., Anderson, J. M. \& Marchant, R. E. (1998). Adhesion of Staphylococcus epidermidis and transposon mutant strains to hydrophobic polyethylene. J Biomed Mater Res 39, $341-350$.

Jacobs, A., Lafolie, F., Herry, J. M. \& Debroux, M. (2007). Kinetic adhesion of bacterial cells to sand: cell surface properties and adhesion rate. Colloids Surf B Biointerfaces 59, 35-45.

Jucker, B. A., Zehnder, A. J. B. \& Harms, H. (1998). Quantification of polymer interactions in bacterial adhesion. Environ Sci Technol 32, 2909-2915.

Katsikogianni, M. \& Missirlis, Y. F. (2004). Concise review of mechanisms of bacterial adhesion to biomaterials and of techniques used in estimating bacteria-material interactions. Eur Cell Mater 8, $37-57$.
Leenaars, A. F. M. \& O'Brien, S. B. G. (1989). Particle removal from silicon substrates using surface-tension forces. Philips J Res 44, 183209.

Lyklema, J. (1991). Electrochemistry and its application to colloids and interfaces. In Fundamentals of Interface and Colloid Science. London: Academic Press.

Meinders, J. M., Van der Mei, H. C. \& Busscher, H. J. (1995). Deposition efficiency and reversibility of bacterial adhesion under flow. J Colloid Interface Sci 176, 329-341.

Mendez-Vilas, A., Gallardo-Moreno, A. M. \& Gonzalez-Martin, M. L. (2006). Nano-mechanical exploration of the surface and sub-surface of hydrated cells of Staphylococcus epidermidis. Antonie Van Leeuwenhoek 89, 373-386.

Mohamed, N., Teeters, M. A., Patti, J. M., Hook, M. \& Ross, J. M. (1999). Inhibition of Staphylococcus aureus adherence to collagen under dynamic conditions. Infect Immun 67, 589-594.

Mohamed, N., Rainier, T. R. \& Ross, J. M. (2000). Novel experimental study of receptor-mediated bacterial adhesion under the influence of fluid shear. Biotechnol Bioeng 68, 628-636.

Norde, W. \& Lyklema, J. (1989). Protein adsorption and bacterial adhesion to solid-surfaces - a colloid-chemical approach. Coll Surf 38, $1-13$.

Ong, Y. L., Razatos, A., Georgiou, G. \& Sharma, M. M. (1999). Adhesion forces between E. coli bacteria and biomaterial surfaces. Langmuir 15, 2719-2725.

Owens, N. F., Gingell, D. \& Rutter, P. R. (1987). Inhibition of celladhesion by a synthetic-polymer adsorbed to glass shown under defined hydrodynamic stress. J Cell Sci 87, 667-675.

Roosjen, A., Boks, N. P., Van der Mei, H. C., Busscher, H. J. \& Norde, W. (2005). Influence of shear on microbial adhesion to PEO-brushes and glass by convective-diffusion and sedimentation in a parallel plate flow chamber. Colloids Surf B Biointerfaces 46, 1-6.

Rutter, P. R. \& Vincent, B. (1988). Attachment mechanisms in the surface growth of microorganisms. In Physiological Models in Microbiology, pp. 87-107. Edited by M. J. Bazin \& J. I. Prosser. Boca Raton, FL: CRC Press.

Sharma, P. K. \& Rao, K. H. (2003). Adhesion of Paenibacillus polymyxa on chalcopyrite and pyrite: surface thermodynamics and extended DLVO theory. Colloids Surf B Biointerfaces 29, 21-38.

Shive, M. S., Hasan, S. M. \& Anderson, J. M. (1999). Shear stress effects on bacterial adhesion, leukocyte adhesion, and leukocyte oxidative capacity on a polyetherurethane. J Biomed Mater Res 46, 511-519.

Simpson, K. H., Bowden, M. G., Hook, M. \& Anvari, B. (2002). Measurement of adhesive forces between S. epidermidis and fibronectin-coated surfaces using optical tweezers. Lasers Surg Med 31, 45-52.

Simpson, K. H., Bowden, A. G., Peacock, S. J., Arya, M., Hook, M. \& Anvari, B. (2004). Adherence of Staphylococcus aureus fibronectin binding protein A mutants: an investigation using optical tweezers. Biomol Eng 21, 105-111.

Thomas, W. E., Nilsson, L. M., Forero, M., Sokurenko, E. V. \& Vogel, V. (2004). Shear-dependent 'stick-and-roll' adhesion of type 1 fimbriated Escherichia coli. Mol Microbiol 53, 1545-1557.

Triandafillu, K., Balazs, D. J., Aronsson, B. O., Descouts, P., Quoc, P. T., Van Delden, C., Mathieu, H. J. \& Harms, H. (2003). Adhesion of Pseudomonas aeruginosa strains to untreated and oxygen-plasma treated poly(vinyl chloride) (PVC) from endotracheal intubation devices. Biomaterials 24, 1507-1518.

Vadillo-Rodriguez, V., Busscher, H. J., Norde, W., De Vries, J. \& Van der Mei, H. C. (2004). Atomic force microscopic corroboration of bond aging for adhesion of Streptococcus thermophilus to solid substrata. J Colloid Interface Sci 278, 251-254. 
Van Holde, K. E. (1971). Introduction in transport processes: diffusion. In Physical Biochemistry, 1st edn, pp. 79-111. Edited by K. E. Van Holde. Englewood Cliffs, NJ: Prentice-Hall.

Van Loosdrecht, M. C. M. \& Zehnder, A. J. B. (1990). Energetics of bacterial adhesion. Experientia 46, 817-822.

Van Oss, C. J. (1994a). Rate of decay with distance. In Interfacial Forces in Aqueous Media, pp. 75-88. Edited by C. J. Van Oss. New York: Marcel Dekker.

Van Oss, C. J. (1994b). Relation between the Hamaker constant and the apolar surface tension component. In Interfacial Forces in Aqueous Media, pp. 154-160. Edited by C. J. Van Oss. New York: Marcel Dekker.

Van Oss, C. J., Good, R. J. \& Chaudhury, M. (1986). The role of van der Waals forces and hydrogen bonds in hydrophobic interactions between biopolymers and low energy surfaces. J Colloid Interface Sci 111, 378-390.

Van Wagenen, R. A. \& Andrade, J. D. (1980). Flat-plate streaming potential investigations - hydrodynamics and electrokinetic equivalency. J Colloid Interface Sci 76, 305-314.

Vijayalakshmi, S. P. \& Raichur, A. M. (2003). The utility of Bacillus subtilis as a bioflocculant for fine coal. Colloids Surf B Biointerfaces 29, 265-275.
Von Eiff, C., Jansen, B., Kohnen, W. \& Becker, K. (2005). Infections associated with medical devices - pathogenesis, management and prophylaxis. Drugs 65, 179-214.

Walker, S. L., Redman, J. A. \& Elimelech, M. (2004). Role of cell surface lipopolysaccharides in Escherichia coli K12 adhesion and transport. Langmuir 20, 7736-7746.

Walt, D. R., Smulow, J. B., Turesky, S. S. \& Hill, R. G. (1985). The effect of gravity on initial microbial adhesion. J Colloid Interface Sci 107, 334-336.

Wang, I. W., Anderson, J. M., Jacobs, M. R. \& Marchant, R. E. (1995). Adhesion of Staphylococcus epidermidis to biomedical polymers contributions of surface thermodynamics and hemodynamic shear conditions. J Biomed Mater Res 29, 485-493.

Xu, L. C., Vadillo-Rodriguez, V. \& Logan, B. E. (2005). Residence time, loading force, $\mathrm{pH}$, and ionic strength affect adhesion forces between colloids and biopolymer-coated surfaces. Langmuir 21, 7491-7500.

Yiantsios, S. G. \& Karabelas, A. J. (1995). Detachment of spherical microparticles adhering on flat surfaces by hydrodynamic-forces. J Colloid Interface Sci 176, 74-85.

Edited by: C. Picioreanu 